\title{
A diagnostic one-step real-time reverse transcription polymerase chain reaction method for accurate detection of influenza virus type $A$
}

\author{
Mohammad Amin Behzadi ${ }^{1,2}$, Mazyar Ziyaeyann ${ }^{1}$, Abdolvahab Alborzi ${ }^{1}$
}

\author{
${ }^{1}$ Professor Alborzi Clinical Microbiology Research Center, Shiraz University of Medical \\ Sciences, Namazi Hospital, Shiraz, Iran \\ ${ }^{2}$ Student Research Committee, Shiraz University of Medical Sciences, Shiraz, Iran
}

Submitted: 27 September 2014

Accepted: 22 November 2014

Arch Med Sci 2016; 12, 6: 1286-1292

DOI: 10.5114 /aoms.2016.62914

Copyright @ 2016 Termedia \& Banach

\section{Abstract}

Introduction: Influenza A is known as a public health concern worldwide. In this study, a novel one-step real-time reverse transcription polymerase chain reaction (rtRT-PCR) assay was designed and optimized for the detection of influenza A viruses.

Material and methods: The primers and probe were designed based on the analysis of 90 matrix nucleotide sequence data of influenza type A subtypes from the GenBank database of the National Center for Biotechnology Information (NCBI). The influenza virus A/Tehran/5652/2010 (H1N1 pdm09) was used as a reference. The rtRT-PCR assay was optimized, compared with that of the World Health Organization (WHO), and its analytical sensitivity, specificity and reproducibility were evaluated. In total, 64 nasopharyngeal swabs from patients with influenza-like illness (ILI) and 41 samples without ILI symptoms were tested for the virus, using conventional cell culture, direct immunofluorescence antibody (DFA) methods, and one-step rtRT-PCR with the designed primer set and probe and the WHO's.

Results: The optimized assay results were similar to the WHO's. The optimized assay results were similar to WHO's, with non-significant differences for $10-10^{3}$ copies of viral RNA/reaction $(p>0.05)$. It detected 10 copies of viral RNA/reaction with high reproducibility and no cross reactivity with other respiratory viruses. A specific cytopathic effect was observed in 6/64 (9.37\%) of the ILI group using conventional culture and DFA staining methods; however, it was not seen in non-ILI. Also, the results of our assay and the WHO's were similar to those of viral isolation and DFA staining.

Conclusions: Given the high specificity, sensitivity and reproducibility of this novel assay, it can serve as a reliable diagnostic tool for the detection of influenza $A$ viruses in clinical specimens and lab experiments.

Key words: influenza A virus, one-step real-time polymerase chain reaction, detection.

\section{Introduction}

Influenza A viruses are one of the major human and animal pathogens, with a global distribution and known as an important public health problem [1, 2]. Previous studies reported that most influenza viruses which have been circulating extensively among the human population since 2003 are of type B and two subtypes H1N1 and H3N2 of type A

\author{
Corresponding author: \\ Mazyar Ziyaeyan PhD \\ Professor Alborzi \\ Clinical Microbiology \\ Research Center \\ Shiraz University \\ of Medical Sciences \\ Namazi Hospital \\ 7193711351 Shiraz, Iran \\ Phone: +98-711 6474304 \\ Fax: +98-711-6474303 \\ E-mail: ziyaeyanm@sums. \\ ac.ir
}


virus $[3,4]$. Despite the availability of flu vaccine and therapeutic agents, annually the disease causes considerable impacts on the life and its quality of at least 500,000 individuals and their families worldwide [5-7]. Accurate and available assays for detection of the infection are not only useful for choosing the best medication procedure after diagnosis, but also help to reduce the rate of antibiotic self-medication for respiratory tract infections [8]. In addition, the emergence of new influenza A variants is highly likely because of antigenic drift, especially antigenic shift in the virus genome sequences [9]. Recent outbreaks caused by pandemic H1N1 2009 [10, 11] and avian-origin influenza H7N9 are striking examples of such threats $[12,13]$. The genetic changes in the viral genome that give the virus the ability to break the species barrier and spread extensively among the human population may result in catastrophic influenza pandemics [14, 15]. The past few outbreaks caused by H5N1 avian influenza virus among humans may potentially be portents of future adverse events $[16,17]$.

To date, a wide range of laboratory assays from traditional culture methods to sophisticated molecular techniques have been applied for accurate diagnosis of the influenza viruses [18]. Poor detection sensitivity, being time-consuming and risky are important factors to take into account when choosing the best assay in clinical and public health laboratories $[19,20]$. Moreover, the constant mutational changes of the viral genome during the evolutionary process may pose a major challenge in developing a universal method or system with the ability to detect different types and strains of the viruses [21]. Thus, novel methods for the detection of influenza viruses circulating in different geographical regions are needed to place under surveillance the viral infections and respective outbreaks in both humans and animals. It was revealed that the matrix $(M)$ gene of the virus is relatively conserved across all influenza A viruses. Therefore, it can be targeted for typing of the virus using molecular methods [22]. In the present study, a novel one-step real-time reverse transcription polymerase chain reaction (rtRT-PCR) assay was designed and optimized for the detection of influenza virus type A. This diagnostic assay was carried out with a set of primers and a labeled TaqMan probe corresponding to the matrix gene of influenza type A viruses.

\section{Material and methods}

\section{Virus strain and culture}

Influenza virus strain A/Tehran/5652/2010 (H1N1 pdm09), as a reference strain, was kindly provided by Dr. T. Mokhtari Azad (School of Pub- lic Health, Tehran University of Medical Sciences). The virus was propagated in Madin-Darby canine kidney (MDCK) cells [23]. The cells were examined on a daily basis for the presence of a specific cytopathic effect (CPE). After the appearance of a CPE, the direct immunofluorescence antibody (DFA) test with specific antibody against influenza A virus nucleoprotein was performed to confirm the presence of the virus, using a commercially available kit (Dakocytomation Ltd; Ely, UK) and according to the manufacturer's instructions. Moreover, nucleic acid was extracted from a $200 \mu$ l volume of cell lysate using a viral RNA isolation kit (Invitek, Berlin, Germany) for further experimentations.

\section{Primer design}

The oligonucleotide primers and probe were designed according to the 90 available $M$ nucleotide sequence data of different subtypes of influenza type A viruses, from the GenBank database of the National Center for Biotechnology Information (NCBI). The subtypes included $20 \mathrm{H} 1 \mathrm{~N} 1$, $20 \mathrm{H} 2 \mathrm{~N} 2,20 \mathrm{H} 3 \mathrm{~N} 2,10 \mathrm{H} 9 \mathrm{~N} 2,10 \mathrm{H} 5 \mathrm{~N} 1$, and 10 H7N3 from all around the world. The sequences were analyzed by the Lasergene sequence analysis software package (DNAStar, Madison, WI, USA), and the primer set and probe were designed based on the conserved sequences. The probe was labeled with 6-carboxyfluorescein (FAM), as a fluorescent reporter dye, and black hole quencher-1 (BHQ-1), at the $5^{\prime}$ and $3^{\prime}$ ends, respectively. Besides cell culture inoculation and DFA, a provided World Health Organization (WHO) rtRT-PCR with the InfA primer-probe set (Table I) was also applied to compare its sensitivity with that of the designed primer and probe set for detecting serially diluted concentrations $\left(10-10^{6}\right)$ of influenza virus type A RNA in three replicates, based on the recommended protocol [24]. All the primer sets and probes were synthesized by AnaSpec (Fremont, CA, USA).

\section{Optimization of one-step real-time polymerase chain reaction}

The reaction was performed using the specific primer set and probe designed to detect influenza virus type $A$. The $25-\mu l$ PCR reaction consists of $12.5 \mu \mathrm{l} 2 \mathrm{X}$ Reaction Mix (Invitrogen, Carlsbad, CA, USA) ), $0.2 \mu \mathrm{M}$ of each primer (FluAF and FluAR), and $0.1 \mu \mathrm{M}$ probe, $0.5 \mu \mathrm{l}$ of SuperScript III Platinum One-Step rtRT-PCR System (Invitrogen, Carlsbad, CA, USA), $0.5 \mu$ l of $25 \mu \mathrm{M}$ ROX reference dye, $5 \mu$ of RNA sample and distilled water. The amplification process was performed in a StepOnePlus Real-Time PCR System instrument (Applied Biosystems, USA), according to the cycling protocol: $50^{\circ} \mathrm{C}$ for $25 \mathrm{~min}$ (reverse transcription) and $95^{\circ} \mathrm{C}$ for $10 \mathrm{~min}$ (DNA polymerase activation), followed 
Table I. Sequences and locations of primers and TaqMan probes used for the detection of influenza virus type A The positions are indicated according to the matrix gene coding domain sequence of influenza $A$ virus ( $A / C a l i f o r-$ nia/04/2009(H1N1)), GenBank accession no.: FJ969513

\begin{tabular}{|lcc|}
\hline Primers and probes & Sequences (5'-3') & Position [bp] \\
\hline $\begin{array}{l}\text { WHO universal influenza A virus forward } \\
\text { primer (InfA Forward) }\end{array}$ & GAC CRA TCC TGT CAC CTC TGA C & $146-167$ \\
\hline $\begin{array}{l}\text { WHO universal influenza A virus reverse } \\
\text { primer (InfA Reverse) }\end{array}$ & AGG GCA TTY TGG ACA AAK CGT CTA & $251-228$ \\
\hline $\begin{array}{l}\text { WHO universal influenza A virus probe } \\
\text { (InfA Probe) }\end{array}$ & (FAM)-TGC AGT CCT CGC TCA CTG GGC ACG-(BHQ1) & $224-201$ \\
\hline FluA forward primer & TCA AAG CCG AGA TCG CRC AGA G & $59-80$ \\
\hline FluA reverse primer & TAG TCA GAG GTG ACA RRA TTG GT & $169-147$ \\
\hline FluA probe & (FAM)-TCT TTA GCC AYT CCA TGA GAG CCT C-(BHQ1) & $142-118$ \\
\hline
\end{tabular}

by 50 cycles of $95^{\circ} \mathrm{C}$ for $15 \mathrm{~s}$ (denaturation) and $55-60^{\circ} \mathrm{C}(55,56,57,58,59,60)$ for $60 \mathrm{~s}$ (annealing and extension).

\section{Construction of the plasmid containing the target $M$ sequence of the influenza virus type $A$}

The designed primer set was used to construct the standard control plasmid containing the target $M$ sequence of the influenza $A$ virus. The extracted RNA was used for the synthesis of the first strand CDNA using M-MuLV reverse transcriptase (Fermentase, Lithuania), and PCR was performed using pfu DNA polymerase (Fermentas, Lithuania) in a $25 \mu$ l reaction. The amplified products were cloned into the $\mathrm{pTZ57R/T}$ and transformed into the Escherichia coli $\mathrm{DH} 5 \alpha$ competent cells (TaKaRa Biotechnology Co., Dalian, China) using a commercially available cloning kit (Fermentase, Lithuania). To confirm the recombinant vectors, positive clones were selected, their plasmids were sequenced with the M13 primers (Shanghai Sangon, China) in the ABI 3130 Genetic Analyzer using the BigDye Terminator v3.1 Cycle Sequencing Kit (Applied Biosystems, USA), and the confirmed plasmid concentration and its copy number per $\mu \mathrm{l}$ of sample were calculated [25]. The resulting plasmid was used as the template for the subsequent analytical studies.

\section{Analytical sensitivity, reproducibility, and specificity}

To detect the sensitivity of the influenza A virus one-step rtRT-PCR method, 10-fold serial dilutions in the range $10^{1}-10^{6}$ copies/ $\mu$ l of the respective recombinant plasmid were prepared and used. To determine the reproducibility of the assay, all the samples were tested in four separate experiments in two replicates of each run for inter- and intra-assay variability. All the mean values, standard deviations (SD) and coefficient of variations
(CV) were calculated using SPSS for Windows software (version 16, SPSS Inc., Chicago, IL, USA). The specificity of the method was tested against influenza virus type $B$, mumps, measles, rubella, Herpes simplex virus 1 and 2, Cytomegalovirus, and Epstein-Barr virus. The primer set and probe were also analyzed using the nucleotide BLAST in the NCBI gene bank to find any potential cross-reactivity with other microorganisms.

\section{Handling and preparation of clinical samples}

A total of 64 nasopharyngeal swabs in viral transport medium from individuals with influenza like illness (ILI) and 41 samples without ILI symptoms were collected in Namazi hospital, southern Iran, during 2011-2012 and sent to the laboratory immediately. Nucleic acid was extracted from a $200 \mu \mathrm{l}$ volume of each sample using a viral RNA isolation kit (Invitek, Berlin, Germany) and used for one-step rtRT-PCR with the designed primer set and probe and those of WHO. In addition, $0.2 \mathrm{ml}$ of each sample was inoculated on the monolayer MDCK cell culture. The cells were daily observed for the CPE and, after a week, lysate of the cell cultures were used for DFA, using a commercially available kit (Dakocytomation Ltd; Ely, UK).

\section{Results}

Optimization of the one-step real-time reverse transcription polymerase chain reaction assay condition

FluAF and FluAR were designed to amplify a $111 \mathrm{bp}$ fragment of the $M$ gene of influenza virus type A. It was successfully cloned into the PTZ57R/T vector. The qRT-PCR was performed optimally in different annealing temperatures $\left(55-60^{\circ} \mathrm{C}\right)$, using our designed primer set and probe, which yielded similar results. As revealed, the results of the optimized one-step TaqMan 
rtRT-PCR were consistent with those from the WHO, when using the same reference RNA sample in serially diluted concentrations (Table II).

Study of the sensitivity, reproducibility, and specificity of the influenza virus type A one-step real-time reverse transcription polymerase chain reaction assay

The sensitivity and reproducibility of the present assay were assessed by serial 10-fold dilutions $\left(10^{1}-10^{6}\right.$ copies $\left./ \mu l\right)$ of the constructed plasmids which were amplified using this one-step rtRT-PCR method in independent and repeated experiments. A standard curve was plotted by amplification of 10 -fold diluted $\left(10^{1}-\right.$ $10^{6}$ copies/ $\left.\mu l\right)$ constructed plasmids versus the threshold cycle (CT) numbers and analysis, using StepOne Software Version 2.1 (Applied Biosystems, Foster City, CA, USA). The detection limit of the target $M$ gene of influenza virus type $A$ was approximately 10 copies per microliter of reaction. The linear regression analysis of the results exhibited a high value of the correlation coefficient $\left(R^{2}=0.997\right)$, indicating the precise and reliable consistency of the replicates (Figure 1 ). The reproducibility of the assay was confirmed
Table II. Detection of 10 -fold dilutions of the influenza virus type A RNA using the designed primer-probe set in this study (FluA), in comparison with those recommended by the WHO $(\operatorname{InfA})$

\begin{tabular}{|lccc|}
\hline \multirow{2}{*}{$\begin{array}{l}\text { Copy } \\
\text { number }\end{array}$} & \multicolumn{2}{c}{$\begin{array}{c}\text { Ct- value (mean } \pm \text { SD) } \\
\text { for indicated assay }\end{array}$} & \\
\cline { 2 - 3 } & FluA & InfA & \\
\hline $10^{6}$ & $15.10 \pm 0.21$ & $16.48 \pm 0.22$ & 0.045 \\
\hline $10^{5}$ & $19.36 \pm 0.18$ & $20.88 \pm 0.23$ & 0.039 \\
\hline $10^{4}$ & $23.29 \pm 0.16$ & $24.90 \pm 0.17$ & 0.021 \\
\hline $10^{3}$ & $27.85 \pm 0.26$ & $28.92 \pm 0.21$ & 0.090 \\
\hline $10^{2}$ & $30.44 \pm 0.29$ & $31.76 \pm 0.22$ & 0.076 \\
\hline $10^{1}$ & $34.97 \pm 0.79$ & $35.10 \pm 0.83$ & 0.920 \\
\hline
\end{tabular}

by high intra-CV and inter-CV as $0.39-1.20 \%$ and $0.38-1.23 \%$, respectively (Table III). The experiments for the evaluation of specificity of the present one-step rtRT-PCR assay with the designed primer set and probe indicated no positive fluorescent signal, when testing the other respiratory viruses. Moreover, based on the blasting search in the NCBI database, no cross reactivity was observed between the respective primer set and probe and other microorganisms' genome sequences.
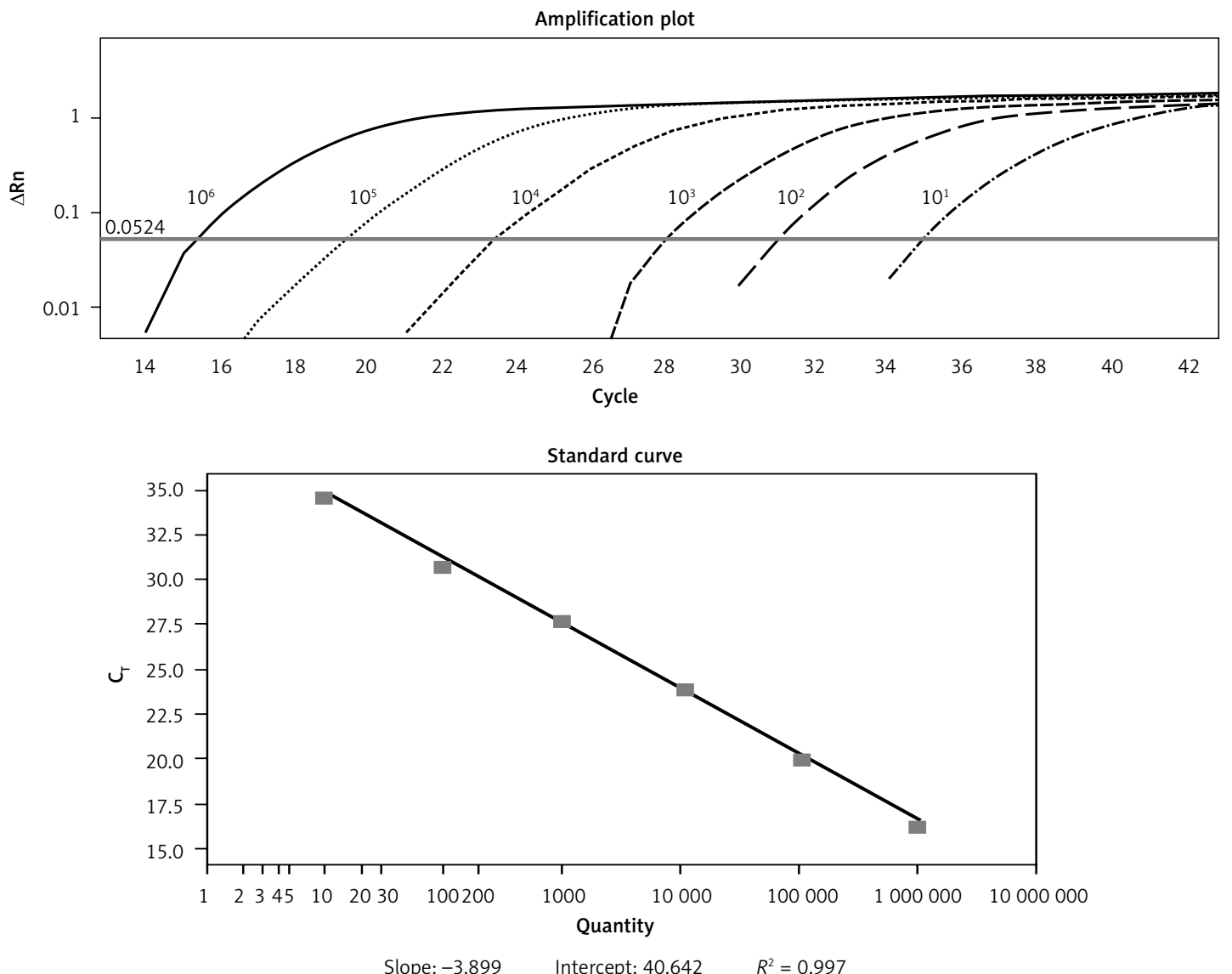

Figure 1. Amplification plot and standard curve of the one-step rtRT-PCR assay for the detection of 10-fold dilutions of the recombinant plasmid containing the target $\mathrm{M}$ gene 
Table III. Intra- and inter-assay coefficients of variation (CV) for detection of influenza virus type $A$ using the designed one-step real-time RT-PCR assay

\begin{tabular}{|lccc|}
\hline Copy number & Mean CT & Intra-CV (\%) & Inter-CV (\%) \\
\hline $10^{6}$ & 15.09 & 0.67 & 0.79 \\
\hline $10^{5}$ & 19.34 & 0.74 & 0.87 \\
\hline $10^{4}$ & 23.18 & 0.43 & 0.70 \\
\hline $10^{3}$ & 27.81 & 0.39 & 0.38 \\
\hline $10^{2}$ & 30.58 & 0.50 & 0.76 \\
\hline $10^{1}$ & 34.60 & 1.20 & 1.23 \\
\hline
\end{tabular}

Evaluating the one-step real-time reverse transcription polymerase chain reaction assay using clinical specimens

Of the 64 nasopharyngeal swabs from patients with ILI, the specific influenza A CPE was observed in $6 / 64(9.37 \%)$ samples; however, it was not detected in non-ILI ones (0/41). The results of DFA staining revealed the same results. Comparison between our one-step rtRT-PCR assay and conventional methods showed that its results were similar to those of viral isolation and DFA staining. In addition, the results of the assay using our primer set and probe and the WHO's were the same (Table IV).

\section{Discussion}

Viral isolation by conventional culture methods currently serves as the gold standard for the detection and typing of influenza viruses, but important limitations including the laborious and time-consuming process, as well as low sensitivity for the detection of some subtypes of the virus, still exist $[26,27]$. Besides, the risk of emergence and human pandemic potential of new influenza A viruses has prompted the attempts to develop more new rapid, sensitive and specific detection methods based on genetic recombination technology and molecular techniques for the most effective management and prevention of the respective infections [28]

In the present study, a novel one-step rtRT-PCR assay was designed to accurately differentiate the influenza virus type A from other respiratory ones. It was demonstrated that the assay was highly sensitive and could achieve a detection limit as low as $10^{1}$ copies per microliter, which was more sensitive than earlier multiplex rtRT-CR assays for simultaneous detection of influenza virus type A and other subtypes or types. A previous study on the simultaneous detection of influenza type $B$ virus and influenza A virus subtypes H5N1,
Table IV. Detection of influenza virus type A RNA in clinical specimens by the designed primer-probe set in this study (FluA), in comparison with those recommended by the WHO (InfA)

\begin{tabular}{|lccc|}
\hline \multirow{2}{*}{$\begin{array}{l}\text { Patient } \\
\text { no. }\end{array}$} & \multicolumn{2}{c}{$\begin{array}{c}\text { Ct- value (mean } \pm \text { SD) } \\
\text { for indicated assay }\end{array}$} & \\
\cline { 2 - 3 } & FluA & InfA & \\
\hline 6 & $28.19 \pm 0.38$ & $29.86 \pm 0.33$ & 0.082 \\
\hline 11 & $15.99 \pm 0.62$ & $17.41 \pm 0.58$ & 0.238 \\
\hline 35 & $21.20 \pm 0.15$ & $22.27 \pm 0.32$ & 0.140 \\
\hline 39 & $22.29 \pm 0.11$ & $23.93 \pm 0.24$ & 0.320 \\
\hline 53 & $19.38 \pm 0.17$ & $20.88 \pm 0.24$ & 0.045 \\
\hline 56 & $15.33 \pm 0.18$ & $16.85 \pm 0.28$ & 0.060 \\
\hline
\end{tabular}

$\mathrm{H} 3 \mathrm{~N} 2$, and H1N1 using a multiplex rtRT-PCR assay showed a sensitivity of approximately $10^{1}-10^{2}$ copies per microliter of reaction [29]. Jiang et al. reported that the sensitivity of the designed rtRT-PCR method for the detection of a novel influenza A (H1N1) virus was 200 copies of in vitro-transcribed target RNA [30]. The sensitivity of a recently developed multiplex rtRT-PCR system for the detection of influenza type $A$ and $B$ viruses and subtype H1N1 by Huber el al. was found to be $3.5 \times 10^{2}$ RNA copies per PCR reaction [31]. However, the detection limit of the present study was almost the same as that of the single rtRTPCR assay for the detection of influenza A virus, studied earlier [32]. The CDC rtRT-PCR Swine Flu Panel has been shown to detect 5 copies of RNA per reaction [33]. Moreover, the results of the present study revealed the same amplification curve in all of the used annealing temperatures $\left(55-60^{\circ} \mathrm{C}\right)$. This may indicate the high efficiency of the designed primers and probe. Also, the current primers and probe were designed based on sequence data at least $10 \%$ of which belong to isolates from Middle East countries including Iraq, Iran, Turkey, Afghanistan, Pakistan, Kuwait, and Qatar. This could increase the specificity of the assay for the detection of domestic viruses circulating in the region.

The efficiency and performance of rtRT-PCR systems are strongly dependent on the quality of the primer sets and probes applied for the amplification of nucleic acid target regions. Development of a rtRT-PCR assay with higher sensitivity and specificity for the detection of influenza $A$ viruses presents a big challenge because of the high mutation frequencies among the sequences of the virus segments. In the present study, oligonucleotide primer set and probe were designed based on the more conserved region of the $M$ segment of the virus genome; however, some degenerative nucleotides were also included. As the results showed, the positivity cycles of the test were similar in all 
used annealing temperatures, indicating the high efficiency of the assay. Moreover, considering the results of inter- and intra-CV, good reproducibility of the test was confirmed. The lack of cross reactivity with other organisms is indicative of high specificity of the test.

Previous studies revealed varying rates of sensitivity for the detection of influenza viruses in clinical specimens using conventional methods. It was reported that the sensitivity of the rapid influenza diagnostic test (RIDT) for the detection of influenza $A(\mathrm{H} 1 \mathrm{~N} 1)$ pdm09 varied between 18\% and $69 \%$. The sensitivity results for DFA were also different and ranged from $47 \%$ to $93 \%$ [34-36]. In the present study, a great similarity was observed between the results of the conventional methods (culture and DFA), our designed assay and those of the WHO. This may be due to the limitation of the number of clinical samples evaluated. The positive results for the assay may increase if more samples are examined, compared with the conventional methods.

In conclusion, given the high specificity, sensitivity and reproducibility of this novel one-step rtRT-PCR, this method can serve as a potential reliable diagnostic tool for the detection of influenza type $A$ both in clinical specimens and lab experiments. However, clinical sensitivity and specificity using more clinical specimens should be evaluated.

\section{Acknowledgments}

Our thanks are due to Hassan Khajehei, for copy editing of the manuscript.

\section{Conflict of interest}

The authors declare no conflict of interest.

\section{References}

1. Cox NJ, Subbarao K. Global epidemiology of influenza: past and present. Annu Rev Med 2000; 51: 407-21.

2. Centers for Disease Control and Prevention. Estimates of deaths associated with seasonal influenza - United States, 1976-2007. MMWR Morb Mortal Wkly Rep 2010; 59: 1057-62.

3. Xu X, Lindstrom SE, Shaw MW, et al. Reassortment and evolution of current human influenza A and B viruses. Virus Res 2004; 103: 55-60.

4. Cohen AL, Hellferscee O, Pretorius M, et al. Epidemiology of influenza virus types and subtypes in South Africa, 2009-2012. Emerg Infect Dis 2014; 20: 1162-9.

5. World Health Organization. Influenza. Fact sheet no. 211. (cited 2005 Apr 25) Available from: http://www. who.int/mediacentre/factsheets/fs211/en/.

6. Heneghan CJ, Onakpoya I, Thompson M, Spencer EA, Jones $M$, Jefferson T. Zanamivir for influenza in adults and children: systematic review of clinical study reports and summary of regulatory comments. BMJ 2014; 348: g2547.

7. Jefferson T, Jones M, Doshi P, Spencer EA, Onakpoya I, Heneghan CJ. Oseltamivir for influenza in adults and children: systematic review of clinical study reports and summary of regulatory comments. BMJ 2014; 348: g2545.

8. Muras M, Krajewski J, Nocun M, Godycki-Cwirko M. A survey of patient behaviours and beliefs regarding antibiotic self-medication for respiratory tract infections in Poland. Arch Med Sci 2013; 9: 854-7.

9. Taubenberger JK, Kash JC. Influenza virus evolution, host adaptation, and pandemic formation. Cell Host Microbe 2010; 7: 440-51.

10. Novel Swine-Origin Influenza A Virus Investigation Team, Dawood FS, Jain S, Finelli L, et al. Emergence of a novel swine-origin influenza A (H1N1) virus in humans. N Engl J Med 2009; 360: 2605-15.

11. Fraser C, Donnelly CA, Cauchemez S, et al. Pandemic potential of a strain of influenza A (H1N1): early findings. Science 2009; 324: 1557-61.

12. Gao R, Cao B, Hu Y, et al. Human infection with a novel avian-origin influenza A (H7N9) virus. N Engl J Med 2013; 368: 1888-97.

13. Ziyaeyan M, Behzadi MA. Novel 2013 influenza A (H7N9) virus: potential pandemic threat. Jundishapur J Microbiol 2013; 6: e11676.

14. Lindh E, Ek-Kommonen C, Väänänen VM, Vaheri A, Vapalahti O, Huovilainen A. Molecular epidemiology of H9N2 influenza viruses in Northern Europe. Vet Microbiol 2014; 172: 548-54.

15. Pascua PN, Choi YK. Zoonotic infections with avian influenza A viruses and vaccine preparedness: a game of "mix and match". Clin Exp Vaccine Res 2014; 3: 140-8.

16. Al-Tawfiq JA, Zumla A, Gautret P, et al. Surveillance for emerging respiratory viruses. Lancet Infect Dis 2014; 14: 992-1000.

17. Maurer-Stroh S, Li Y, Bastien N, et al. Potential human adaptation mutation of Influenza A(H5N1) Virus, Canada. Emerg Infect Dis 2014; 20: 1580-2.

18. Kim DK, Poudel B. Tools to detect influenza virus. Yonsei Med J 2013; 54: 560-6.

19. Lucas PM, Morgan OW, Gibbons TF, et al. Diagnosis of 2009 pandemic influenza A (pH1N1) and seasonal influenza using rapid influenza antigen tests, San Antonio, Texas, April-June 2009. Clin Infect Dis 2011; 52: S116-22.

20. Kiilerich-Pedersen K, Daprà J, Cherré S, Rozlosnik N. High sensitivity point-of-care device for direct virus diagnostics. Biosens Bioelectron 2013; 49: 374-9.

21. Yang Z, Mao G, Liu Y, et al. Detection of the pandemic H1N1/2009 influenza A virus by a highly sensitive quantitative real-time reverse-transcription polymerase chain reaction assay. Virol Sin 2013; 28: 24-35.

22. Furuse Y, Suzuki A, Kamigaki T, Oshitani H. Evolution of the $M$ gene of the influenza $A$ virus in different host species: large-scale sequence analysis. Virol J 2009; 6: 67.

23. Balish AL, Katz JM, Klimov AI. Influenza: propagation, quantification, and storage. Curr Protoc Microbiol 2013; 15G-1.

24. World Health Organization. 2009. CDC protocol of real-time RT-PCR for swine influenza (H1N1). World Health Organization, Geneva, Switzerland. http://www.who.int/ csr/resources/publications/swineflu/CDCrealtimeRTPCRprotocol 20090428.pdf. Accessed 8 May 2009.

25. Lv J, Wei B, Chai T, et al. Development of a real-time RTPCR method forrapid detection of H9 avian influenza virus in the air. Arch Virol 2011; 156: 1795-801.

26. Templeton KE, Scheltinga SA, Beersma MF, Kroes AC, Claas EC. Rapid and sensitive method using multiplex real-time PCR for diagnosis of infections by influenza $A$ and influenza $B$ viruses, respiratory syncytial virus, and 
parainfluenza viruses $1,2,3$, and 4. J Clin Microbiol 2004; 42: 1564-9.

27. Sidoti F, Rizzo F, Costa C, et al. Development of real time RT-PCR assays for detection of type $A$ influenza virus and for subtyping of avian $\mathrm{H} 5$ and $\mathrm{H} 7$ hemagglutinin subtypes. Mol Biotechnol 2010; 44: 41-50.

28. Behzadi MA, Hosseini A, Dadras H. Cloning and bacterial expression of hemagglutinin antigen stem of $\mathrm{H} 9 \mathrm{~N} 2$ avian influenza virus. Int J Curr Microbiol App Sci 2014; 3: 112-9.

29. Shisong F, Jianxiong L, Xiaowen C, et al. Simultaneous detection of influenza virus type $B$ and influenza $A$ virus subtypes $\mathrm{H} 1 \mathrm{~N} 1, \mathrm{H} 3 \mathrm{~N} 2$, and $\mathrm{H} 5 \mathrm{~N} 1$ using multiplex real-time RT-PCR. Appl Microbiol Biotechnol 2011; 90: 1463-70.

30. Jiang T, Kang X, Deng Y, et al. Development of a real-time RT-PCR assay for a novel influenza A (H1N1) virus. J Virol Methods 2010; 163: 470-3.

31. Huber I, Campe H, Sebah D, et al. A multiplex one-step real-time RT-PCR assay for influenza surveillance. Euro Surveill 2011; 16: pii:19798.

32. Panning M, Baumgarte $S$, Laue T, et al. Singleplex real-time RT-PCR for detection of influenza A virus and simultaneous differentiation of $\mathrm{A} / \mathrm{H} 1 \mathrm{~N} 1 \mathrm{v}$ and evaluation of the RealStar influenza kit. J Clin Virol 2011; 50: 171-4.

33. Shu B, Wu KH, Emery S, et al. Design and performance of the $C D C$ real time reverse transcriptase PCR swine flupanel for detection of 2009 A (H1N1) pandemic influenza virus. J Clin Microbiol 2011; 49: 2614-9.

34. Nutter S, Cheung M, Adler-Shohet FC, Krusel K, Vogel K, Meyers $\mathrm{H}$. Evaluation of indirect fluorescent antibody assays compared to rapid influenza diagnostic tests for the detection of pandemic influenza A (H1N1) pdm09. PLoS One 2012; 7: e33097.

35. Ganzenmueller T, Kluba J, Hilfrich B, et al. Comparison of the performance of direct fluorescent antibodystaining, a point-of-care rapid antigen test and virus isolation with that of RT-PCR for the detection of novel 2009 influenza $A(H 1 N 1)$ virus in respiratory specimens. J Med Microbiol 2010; 59: 713-7.

36. Sandora TJ, Smole SC, Lee GM, Chung S, Williams L, McAdam AJ. Test characteristics of commercial influenza assays for detecting pandemic influenza $A(H 1 N 1)$ in children. Pediatr Infect Dis J 2010; 29: 261-2. 